die beiden dem Methylen benachbarten Kohlenstoffatome sich so nahe kommen, dass zwischen ihnen ein theilweiser Ausgleich der Partialvalenzen stattfindet. Ein solcher Körper wäre noch ungesättigt, könnte in Benzolderivate übergehen und die Energie der endständigen Kohlenstoffatome wäre doch nicht gross genug, um die Methylenwasserstoffatome so reactionsfähig zu machen, wie im Cyklopentadiën. Es scheint mir das ein Ausweg, um die auch von Willstätter betonten Unterschiede zwischen Tropiliden und Cyklopentadiën verständlich zu machen.

Bei diesen Versuchen erfrente ich mich der ausgezeichneten Unterstützung des Herrn Dr. F. Straus.

Ueber die Einwirkung der salpetrigen und der Salpetersäure auf das Mesityloxydoxim;

von C. Harries.

(Aus dem I. Berliner Universitiitslaboratorium.)

(Eingelaufen am 10. October 1901.)

In der vorliegenden Abhandlung habe ich eine Anzahl von Verbindungen beschrieben, welche Einwirkungsproducte der salpetrigen und der Salpetersäure auf das Mesityloxim sind oder sich von diesen ableiten. Die mannigfaltigen Umwandlungen zeigen, wie reactionsfähig die Oximidogruppe ist, wenn sie sich in Nachbarschaft zu einer Kohlenstoffdoppelbindung befindet.

Die einzelnen Reactionen sind möglichst durchgearbeitet worden, um eventuell einen neuen Weg aufufinden, Oximidoverbindungen, wie man sie aus hochmolekularen Substanzen durch Einwirkung von Salpetersäure oder salpetriger Säure oftmals erhält, nach einer glatten Methode zu Körpern einfacherer Molekulargrösse abzubauen.

Ein ähulicher Gedankengang ist übrigens schon von anderer Seite früher verfolgt worden. So von Tiemann und Mahla 
beim Kampher- und Fenchonoxim und von Wallach einerseits und Semmler andererseits beim Terpinennitrosit. Auch der Untersuchung Pesci's über das Phellandrennitrit, welche von Wallach ${ }^{1}$ ) fortgeführt wurde, muss hier gedacht werden.

Inwieweit es mir gelungen ist, diese Absicht za verwirklichen, mag an einem Beispiele illustrirt werden. Das Mesityloxyd lässt sich auf solchem Wege bequem in Nitroaceton überführen :

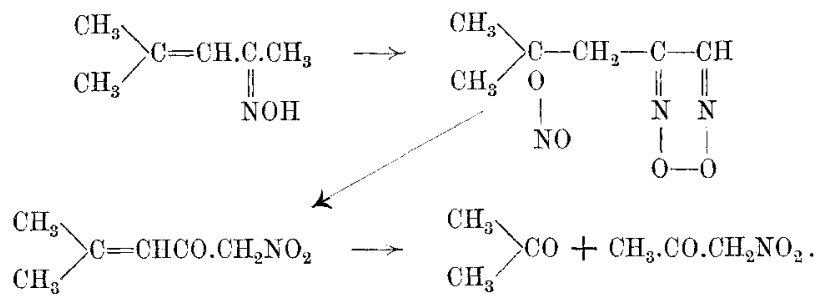

Man gelangt also hiernach zu Körpern der halben Molekulargrösse. In der Mesityloxydreihe wurde mir der Versuch dadurch sehr erleichtert, dass die betreffenden Substanzen oder ihre Derivate fast sämmtlich hervorragend schön krystallisiren und die Uebergänge sich mit geringer Ausnabme fast quantitativ vollziehen. Ich weise aber darauf hin, dass auch in anderen Reihen diese Methode Anwendung finden wird, wenn man sie etwas modificirt und hoffe mich ibrer später mit Erfolg für Constitutionsaufklärungen bedienen zu können ${ }^{2}$ ).

\section{Einwirkung der salpetrigen Säure auf Mesityloxydoxim.}

Löst man die stereomeren Oxime ${ }^{3}$ ) des Mesityloxyds in verdünnten Mineralsäuren und fügt Natriumnitrit hinzu, so erhält man das sogenannte, schon früher ausführlich beschriebene ${ }^{4}$ ) Mesitylnitrimin. In quantitativer Ausbente gewinnt man diesen

\footnotetext{
1) Diese Annalen 287, 376 (1895); 313, 345 (1900).

2) Vergl. Ber. d. deutsch. chem. Ges. 34, 2291 (1901).

3) Harries und Jablonski, Ber. d. deutsch. chem. Ges. B1, 1371 (1898).

4) Harries und Gley, Ber. d. deutsch. chem. Ges. B2, 1330 (1899).
} 
Körper beim Vermischen gleicher Theile des $\alpha$-oder alkalistabilen Mesityloxims mit Eisessig und Isoamylnitrit.

In Bezug auf die Eigenschaften des Mesitylnitrimins ist noch Folgendes nachzutragen. Durch zehn Minuten langes Kochen mit 40procentiger Kalilauge wird dasselbe rückwärts zerlegt in $\alpha$-Mesityloxim und salpetrigsaures Kalium. Hieraus, wie aus dem Verhalten gegen Brom und Permanganat geht die ungesättigte Natur der Verbindung zur Genüge hervor. Brom in Eisessig wird nach einigem Stehen allmählich addirt, durch Wasser wird aus dem Reactionsproducte ein bromhaltiger, weisser Körper gewonnen. Wässrige Permanganatlösung wird sofort unter Abscheidung von Braunstein entfärbt, es scheint dabei ein vollständiger Zerfall des Moleküls einzutreten.

Verbindungen von ähnlichem Typus sind bereits von mehreren Chemikern dargestellt worden, so beim Kampheroxim von Angeli und Rimini ${ }^{5}$ ), Tiemann und Mahla $\left.{ }^{6}\right)$. Letztere fassen das Salpetrigsäurederivat des Kampheroxims als ein Nitramid auf, wegen seiner Fähigkeit, sich in Alkali unter Salzbildung zu lösen. Unter Zugrundelegung der Bredt'schen Kampherformel würde es folgendermassen zu formuliren sein:

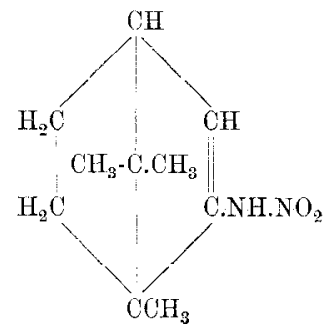

Da das Salpetrigsäurederivat des Mesityloxims diese Eigenschaft nicht anzeigt, wurde seine Formel derjenigen des Pinakolinnitrimins ${ }^{7}$ ), dem Einwirkungsproducte des Stickstofftetroxyd

5) Gazz. chim. 25, [1] 406 (1895); 26, [1] 29, 34 (1896).

6) Ber. d. deutsch. chem. Ges. 28, 1079 (1895); 29, 2807 (1896).

7) Scholl and Born, Ber. d. deutsch. chem. Ges. 28, 1361 (1895). 
Uebersicht der dargestellten Verbindungen und ihr genetischer Zusammenhang.

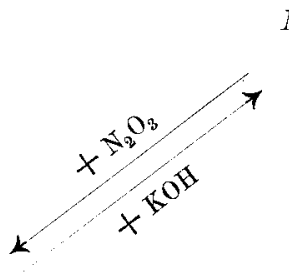

Mesityloxydoxim

$\mathrm{C}_{6} \mathrm{H}_{11} \mathrm{NO}$

$\mathrm{C}=\mathrm{CH} . \mathrm{C} . \mathrm{CH}$

$\mathrm{H}_{3}$

HON $\quad x+40$

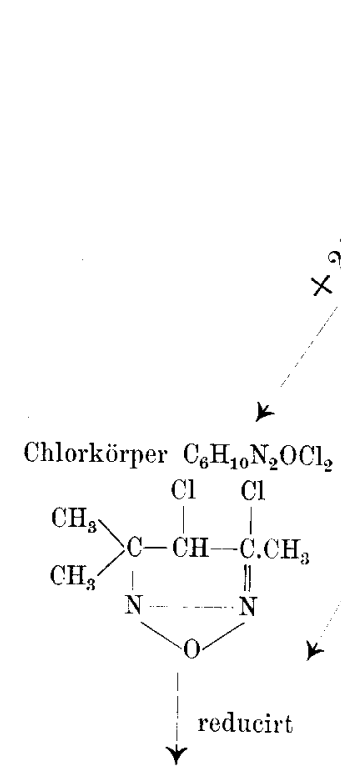

Trimethyldihydropyrazolin

$$
\mathrm{CH}_{3}
$$

$\mathrm{CH}_{3} \mathrm{CH}=\mathrm{C} \cdot \mathrm{CH}_{3}$

\section{Mesityluitrimin}

$$
\mathrm{C}_{6} \mathrm{H}_{10} \mathrm{~N}_{2} \mathrm{O}_{2}
$$

$\stackrel{\mathrm{CH}_{3}}{\mathrm{CH}_{8}, \mathrm{C}=\mathrm{CH} . \mathrm{C}-\mathrm{CH}_{3}}$

ó

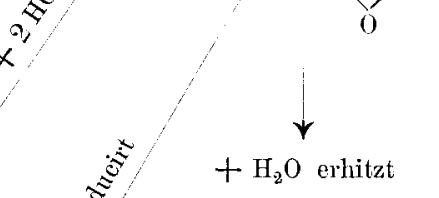

Isomesitylnitrimin

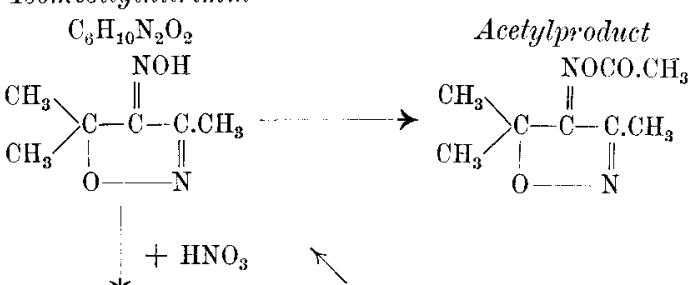

Körper $\mathrm{C}_{6} \mathrm{H}_{9} \mathrm{~N}_{8} \mathrm{O}_{5}$

$$
\checkmark \text { erhitzt }
$$

Trimethyldihydroketoisoxazol $\mathrm{O}_{9} \mathrm{H}_{9} \mathrm{NO}_{2}$

$\mathrm{CH}_{3}$

$$
\begin{aligned}
& { }^{\mathrm{CH}_{3}}>{ }_{\mathrm{O}}^{\mathrm{C}-\mathrm{CO}-\|_{N}^{\mathrm{C}} \cdot \mathrm{CH}_{3}}+\mathrm{H}_{2} \mathrm{NOH} \\
& \text { + Phenyl- } \\
& \downarrow \text { hydrazin } \\
& \mathrm{CH}_{3} \backslash \mathrm{CH}_{3} \overbrace{0}^{\mathrm{N}-\mathrm{NHC}_{6} \mathrm{H}_{3}}
\end{aligned}
$$

+ Anilin in Eisessig.

$$
+ \text { Anilin in Aether }
$$

Anil- $\alpha$-nitromesityloxyd

$$
\begin{gathered}
\mathrm{CH}_{3} \backslash \underset{\mathrm{H}_{3}}{\mathrm{C}=}=\underset{\|_{\mathrm{NC}_{6} \mathrm{H}_{5}} \mathrm{CH}_{14} \mathrm{~N}_{2} \mathrm{O}_{2}}{\mathrm{CH}_{2} \mathrm{NO}_{2}} \\
+\mathrm{HCl} \\
+
\end{gathered}
$$

c-Nitromesityloxyd

$$
\mathrm{C}_{6} \mathrm{H}_{9} \mathrm{NO}_{3}
$$

$\mathrm{CH}_{3}>\mathrm{C}=\mathrm{CHCO}_{3} \mathrm{CH}_{2} \mathrm{NO}_{2}$
Semicarbazon $\mathrm{CH}_{3}$. C.C.CH $\mathrm{NO}_{2}$ N.NH.CO. $\mathrm{NH}_{2}$

\section{Nitroaceton \\ $\mathrm{C}_{3} \mathrm{H}_{\overline{5}} \mathrm{NO}_{3}$

$$
\mathrm{CH}_{3} \cdot \mathrm{CO} . \mathrm{CH}_{2} \mathrm{NO}_{2}
$$

$$
+ \text { Benz- }
$$$$
\downarrow \text { aldehyd }
$$

a-Nitrobenzalaceton $\mathrm{C}_{10} \mathrm{H}_{9} \mathrm{NO}_{3}$

$\mathrm{C}_{6} \mathrm{H}_{5} \mathrm{CH}=\mathrm{CH} . \mathrm{CO} . \mathrm{CH}_{2} \mathrm{NO}_{2}$ 
auf eine ätherische Pinakolinoximlösung, nachgebildet und die Bezeichnung Mesitylnitrimin gewählt:<smiles>CC(C)=CC(C)NOC(C)(C)C(C)(C)C</smiles>

Ich habe nun eine Reihe neuer Versuche angestellt, welche im Wesentlichen die Richtigkeit dieser Constitution bestätigen und nur eine unbedeutende Modification derselben nöthig erscheinen lassen.

Durch Reduction mit Zinkstaub und Wasser wird das Mesitylnitrimin nämlich leicht in ein Iydrazinderivat, das schon von Ourtius und Wirsing ${ }^{8}$ ) dargestellte Trimethyldihydropyrazolin übergeführt:<smiles>CC1=NNC(C)(C)C1</smiles>

Hieraus geht hervor, dass die beiden Stickstoffatome im Mesitylnitrimin direct aneinander gekettet sind und nicht etwa ein Oximsalpetrigsäureester, wie ich früher vermuthete ${ }^{9}$ ), vorliegt.

Reduction des Mesitylnitrimins mit Zinlstaub und Wasser.

$5 \mathrm{~g}$ Nitrimin werden mit $15 \mathrm{~g}$ Zinkstaub und $100 \mathrm{ccm}$ Wasser unter Rückfluss sechs Stunden gekocht, die entstandene Base wird nach dem Abfiltriren des Zinkstaubes durch Natriumcarbonat abgeschieden, mit Aether aufgenommen und aus der ätherischen Flüssigkeit mittelst einer Auflösung von wasserfreier Oxalsäure in absolutem Aether als Oxalat ausgefällt. Dasselbe bildet, aus absolutem Alkohol umkrystallisirt, eine weisse Krystallmasse, die bei $147-148^{\circ}$ schmilzt.

8) Journ. f. pract. Chem. 50, 546 (1894).

9) Harries, Ber. d. dentsch. chem. Ges. 33, 1999 (1900). 
234 Harries, Ueber die Einwirkung der salpetrigen und der

I. $0,1656 \mathrm{~g}$, vacuumtrocken, gaben $0,2886 \mathrm{CO}_{2}$ und $0,1059 \mathrm{H}_{2} \mathrm{O}$. $0,1912 \mathrm{~g}$ gaben $0,3334 \mathrm{CO}_{2}$ and $0,1246 \mathrm{H}_{2} \mathrm{O}$.

II. $0,1688 \mathrm{~g} \quad, \quad 21 \mathrm{ccm}$ Stickgas bei $24^{\circ}$ und $762 \mathrm{~mm}$ Druck.

\begin{tabular}{ccrr} 
& Berechnet für & $\overbrace{3}^{\text {Gefunden }}$ \\
$\mathrm{C}$ & $\mathrm{C}_{6} \mathrm{H}_{12} \mathrm{~N}_{2}\left(\mathrm{CO} \mathrm{O}_{3} \mathrm{H}\right)_{2}$ & \multicolumn{1}{l}{$\mathrm{II}}$. \\
$\mathrm{H}$ & 47,52 & 47,53 & 47,56 \\
$\mathrm{~N}$ & 6,93 & 7,11 & 7,24 \\
$\mathrm{~N}$ & 13,86 & - & 14,00
\end{tabular}

Das Trimethyldihydropyrazolin wird in reinem Zustande aus dem Oxalat durch Lösen in Wasser und Abscheiden mit Kaliumcarbonat gewonnen.

Im Vacuum siedet die Base bei $63-64^{\circ}$ unter $23 \mathrm{~mm}$ Druck und bildet ein stark lichtbrechendes, wasserhelles Liquidum von charakteristischem, pyrazolähnlichem Geruch. Sie ist in jeder Beziehung identisch mit der aus Mesityloxyd und Hydrazinhydrat darstellbaren Verbindung. Nur füge ich hinzu, dass sie beim Kochen etwas Fehling'sche Lösung reducirt.

$0,1363 \mathrm{~g}$ gaben $0,3187 \mathrm{CO}_{2}$ und $0,1353 \mathrm{H}_{2} \mathrm{O}$.

$\begin{array}{ccc} & \text { Berechnet für } & \text { Gefunden } \\ & \mathrm{C}_{6} \mathrm{H}_{12} \mathrm{~N}_{2} & \\ \mathrm{C} & 64,29 & 63,65 \\ \mathrm{H} & 10,71 & 11,03\end{array}$

Das Chlorhydrat wird erhalten durch Einleiten von Salzsäuregas in eine absolut ätherische Lösung der Base. Es fällt zuerst als Oel aus, welches allmählich beim Reiben krystallisirt. Durch Aufnehmen in absolutem Alkohol und Ausfällen mit absolutem Aether wird es in analysenreine Form gebracht und schmilzt dann bei $176-177^{\circ}$.

$0,2035 \mathrm{~g}$, vacuumtrocken, gaben $0,3614 \mathrm{CO}_{2}$ und $0,1615 \mathrm{H}_{2} \mathrm{O}$.

$0,15559 \mathrm{~g}$ gaben $0,1513 \mathrm{AgCl}$.

Berechnet für $\mathrm{O}_{6} \mathrm{H}_{12} \mathrm{~N}_{2} \mathrm{ClH}$

C

H

$\mathrm{Cl}$
48,48

8,75

23,91
Gefunden

48,44

8,82

23,99 
Früher ${ }^{10}$ ) ist ein Product der Einwirkung von Salzsäure auf das Mesitylnitrimin beschrieben worden, welches sehr schön in perlmutterartig glänzenden Blättern krystallisirt und in Anbetracht seiner äusserst stabilen Eigenschaften als eine ringförmige Verbindung angesprochen wurde.

Dasselbe entsteht nach folgender Gleichung:

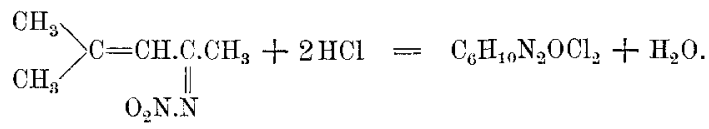

Man sieht, dass hierbei eine partielle Reduction des Mesitylnitrimins erfolgt ist and ich glaube, dass der chlorhaltige Körper $\mathrm{C}_{6} \mathrm{H}_{10} \mathrm{~N}_{2} \mathrm{OCl}_{2}$ eine Art von Zwischenglied bei der Reduction des Mesitylnitrimin zum Trimethyldihydropyrazolin bildet. Denn das chlorhaltige Product liefert mit Zinkstaub und Wasser behandelt glatt ebenfalls das Trimethyldihydropyrazolin.

Man wird also wohl nicht fehl gehen, wenn man den Chlorkörper bereits als ein Pyrazolinderivat ansieht und annimmt, dass der Sauerstoff, da er so leicht herausgenommen wird, am Stickstoff gebunden ist. Schwierigkeiten macht nur die Vertheilung der Chloratome, für deren Lagerung mehrere Möglichkeiten gegeben sind. Ich nehme zunächst Ringschliessung unter Verschiebung der Doppelbindung an, wobei folgendes Zwischenproduct entsteht:

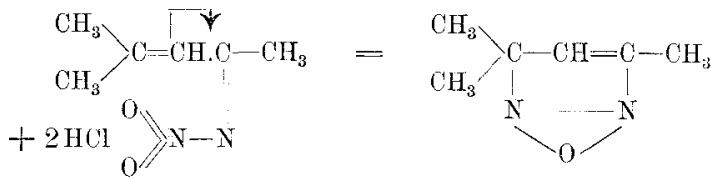

Zwischenproduct.

An diese Doppelbindung Iagern sich dann die Chloratome:

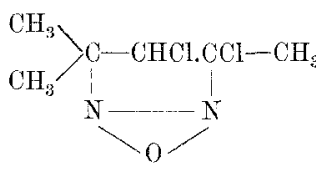

10) Ifarries und Gley, Ber. d. deutsch. chem. Ges. 32, 1337 (1899).<smiles>CC=CC(C)(C)N</smiles>

Trimethyldihydropyrazolin. 
Einfacher lässt sich der Uebergang des Mesitylnitrimins in den Chlorkörper darstellen, wenn man dasselbe nicht nach der eben gegebenen, sondern folgendermassen formulirt,

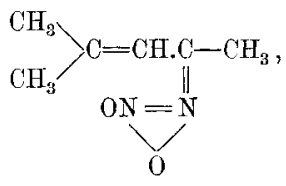

d. h. der Nitrimingruppe zwei fünfwertbige Stickstoffatome beilegt.

Reduction des Chlorkörpers mit Zinkstawb und Wasser.

$10 \mathrm{~g}$ Chlorkörper $\mathrm{C}_{6} \mathrm{H}_{10} \mathrm{~N}_{2} \mathrm{OCl}_{2}$ werden mit $30 \mathrm{~g}$ Zinkstaub und $250 \mathrm{~g}$ Wasser unter Rückfluss sechs Stunden gekocht. Die vom Zinkstaub abfiltrirte Flüssigkeit wird mit starker Natronlauge versetzt und die abgeschiedene Base mit Wasserdampf übergetrieben. Das Destillat wird mit Kaliumcarbonat gesättigt, wiederholt ansgeäthert und nach dem Verdampfen des Aethers der hinterbleibende, ölige Rückstand im Vacuum fractionirt. Die grösste Menge desselben siedet zwischen $40^{\circ}$ und $90^{\circ}$. Durch eine absolut ätherische Lösung von wasserfreier Oxalsäure wird hieraus das vorhin beschriebene Oxalat des Trimethyldihydropyrazolins isolirt, welches nach dem Umkrystallisiren aus absolutem Alkohol bei $147-148^{\circ}$ schmilzt. Ausbeute etwa $7 \mathrm{~g}$ Oxalat.

Verhalten des Mesitylnitrimins beim Erhitzen mit Wasser. Isomesitylnitrimin.

Ein recht eigenthümliches Verhalten zeigt das Mesitylnitrimin, wenn es mit Wasser im Einschlussrohre zwei Stunden auf $120^{\circ}$ erhitzt wird. Es entsteht hierbei ein neuer Körper, welcher dem Mesitylnitrimin, $\mathrm{C}_{6} \mathrm{H}_{10} \mathrm{~N}_{2} \mathrm{O}_{2}$, isomer, aber structurverschieden davon ist.

Da bei dieser Umlagerung des Mesitylnitrimins in Isomesitylnitrimin, wie ich vorweg nehmen will, das Oxim eines cyklischen Ketons der Formel $\mathrm{C}_{6} \mathrm{H}_{9} \mathrm{NO}_{2}$ entsteht, so ist dieselbe nur im Sinne folgender Gleichungen zu deuten: 


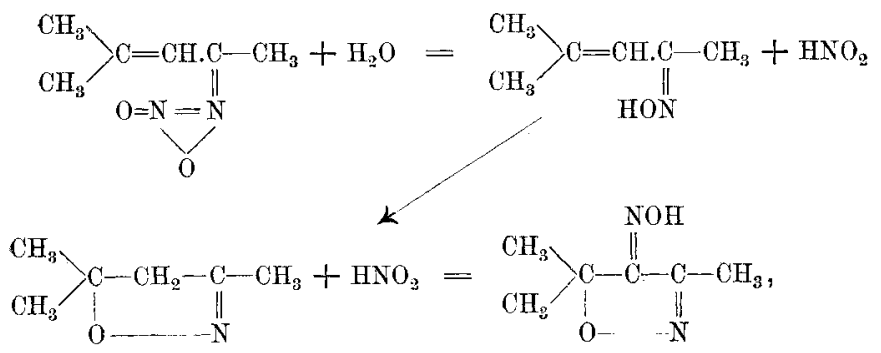

d. h. es bildet sich das Oxim des Ketotrimethyldihydroisoxazols, für das ich der Einfachheit halber den Namen Isomesitylnitrimin beibehalte.

Die Ausbeute beträgt etwa $90 \mathrm{pC}$. Aus Chloroform umkrystallisirt, bildet es rein weisse Blätter, welche bei $156^{\circ}$ bis $157^{\circ}$ schmelzen, während sich das Mesitylnitrimin bei derselben Temperatur zersetzt.

$0,1809 \mathrm{~g}$, vacuumtrocken, gaben $30,7 \mathrm{ccm}$ Stickgas bei $14^{0}$ und $758 \mathrm{~mm}$ Druck.

$0,2008 \mathrm{~g}^{*}$ gaben $0,3731 \mathrm{CO}_{2}$ und $0,1249 \mathrm{H}_{2} \mathrm{O}$.

$\begin{array}{ccr} & \text { Berechnet für } & \text { Gefunden } \\ & \mathrm{C}_{6} \mathrm{H}_{10} \mathrm{~N}_{2} \mathrm{O}_{2} & \\ \mathrm{C} & 50,68 & 50,70 \\ \mathrm{H} & 6,91 & 7,04 \\ \mathrm{~N} & 20,01 & 19,72\end{array}$

$0,2243 \mathrm{~g}$, in $27 \mathrm{~g}$ Eisessig gelöst, gaben eine Depression von 0,207".

$\begin{array}{ccc} & \text { Berechnet } & \text { Gefunden } \\ \text { Nolgew. } & 142 & 157\end{array}$

Das Isomesitylnitrimin besitzt die Eigenschaften eines Oxims, es löst sich in Mineralsäuren und Alkalien. Es bildet mit Säuren keine krystallinischen Salze, wohl aber eine Natriumverbindung, welche man durch Lösen in der berechneten Menge Natriumalkoholat und Fällen mit Aether erhält. Der Körper reducirt an und für sich bein Kochen nicht Fehlingsche Lösung. Erhitzt man aber erst mit verdünnter Schwefelsäure und neutralisirt dann mit Alkali, so ist das abgespaltene Ilydroxylamin durch Fehling'sche Flüssigkeit leicht nachzuweisen. Es enthält keine Kohlenstoffdoppelbindung, da weder 
in Eisessig noch in Schwefelkohlenstoff Brom addirt wird. Durch Reduction mit Zinkstaub und Wasser tritt keine Veränderung ein. Während das Mesitylnitrimin durch Essigsäureanbydrid nicht verändert wird, liefert das Isonitrimin leicht damit ein Acetylderivat.

Das Acetylisomesitylnitrimin wird durch Lösen in überschüssigem Essigsäureanhydrid und durch Eindampfen auf dem Wasserbade als krystallinische Masse gewonnen. Durch Aufstreichen auf Thon und Umkrystallisiren aus absolutem Alkohol oder Petroläther wird es gereinigt und besitzt dann den Schmelzpunkt $68-69^{\circ}$.

$0,1672 \mathrm{~g}$ gaben $21,8 \mathrm{ccm}$ Stickgas bei $16^{\circ}$ und $750 \mathrm{~mm}$ Druck.

$0,1682 \mathrm{~g} \quad, \quad 0,3216 \mathrm{CO}_{2}$ und $0,1008 \mathrm{H}_{2} \mathrm{O}$.

$\begin{array}{ccr} & \text { Berechnet für } & \text { Gefunden } \\ & \mathrm{O}_{6} \mathrm{H}_{9} \mathrm{~N}_{2} \mathrm{O}_{2}\left(0 \mathrm{C} \cdot \mathrm{CH} \mathrm{H}_{3}\right) & \\ \mathrm{C} & 52,15 & 52,17 \\ \mathrm{H} & 6,66 & 6,52 \\ \mathrm{~N} & 15,11 & 15,22\end{array}$

Durch Einwirkung von Benzoylchlorid auf das Natriumsalz des Isomesitylnitrimins bildet sich auch leicht ein Benzoylderivat.

Darstellung des dem Isomesitylnitrimin zu Grunde liegenden Ketons, Ketotrimethyldihydroisaxazol.

Dio Oximidogruppe des Isomesitylnitrimins lässt sich durch Kochen mit verdünnten Säuren nur unter grossem Verlust an Substanz abspalten. Es entsteht hierbei unter Zerfall des ganzen Moleküls etwas Acetoxim. Folgender Weg hat dagegen glatt zum Ziele geführt:

Das Isomesitylnitrimin wird durch Uebergiessen mit concentrirter Salpetersäure in ein Oel umgewandelt, welches die Formel $\mathrm{C}_{6} \mathrm{H}_{9} \mathrm{~N}_{3} \mathrm{O}_{5}$ besitzt und ein Salpetersäurederivat von ersterem ist. Die Constitution dieses Körpers konnte nicht genau aufgeliärt werden, ist aber auch zunächst gleichgültig, weil es seinen Zweck als Zwischenglied zur Darstellung des Ketotrimethyldihydroisoxazols zu dienen erfüllt hat. Beim Erhitzen 
für sich oder mit Essigsäureanhydrid verliert es nämlich salpetrige Säure und liefert dieses Keton, welches durch Hydroxylamin wieder in das Isomesitylnitrimin zurückverwandelt wird. Vielleicht lässt sich der Vorgang in folgenden Formeln deuten:

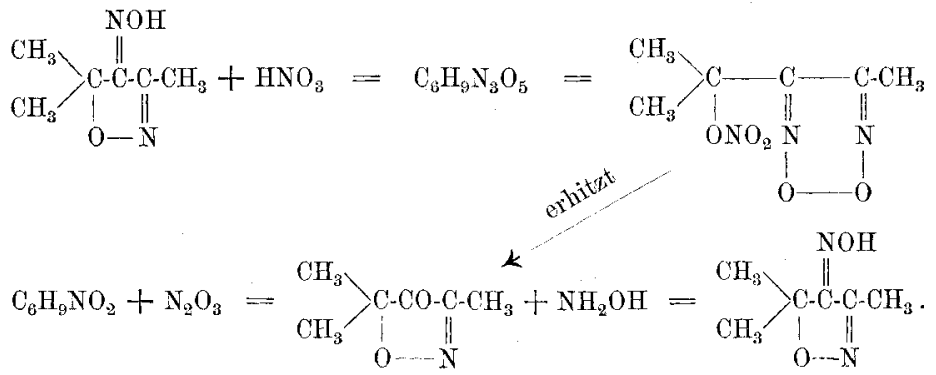

Es bildet sich darnach unter Aufspaltung des Isoxazolringes Mesitylnitrosatglyoximhyperoxyd, dessen Entstehung mir unter Berücksichtigung der später geschilderten Eigenschaften des Mesitylnitritglyoximhyperoxyds nicht so unwahrscheinlich erscheint. Auch das Verhalten dieses Körpers gegenüber Natriummethylat, wodurch Natriumnitrat abgespalten wird, zeigt, dass eine nitrosatähnliche Verbindung vorliegt ${ }^{11}$ ).

$5 \mathrm{~g}$ Isomesitylnitrimin werden unter Kühlung mit $20 \mathrm{ccm}$ Salpetersäure vom spec. Gew. 1,40 übergossen. Nach etwa zwei Stunden lebhafter Entwickelung brauner Dämpfe hat sich ein gelbes, dickes, kampherartig riechendes Oel gebildet, welches von der Salpetersäure abgehoben und bis zur neutralen Reaction mit Soda, verdünnter Natronlauge und Wasser gewaschen wird. Das Oel wurde zur Analyse nur mit Calciumchlorid getrocknet, da es sich bei der Destillation auch im Vacuum zersetzt. Die Ausbeute ist quantitativ.

$0,1490 \mathrm{~g}$ gaben $0,1951 \mathrm{CO}_{2}$ und $0,0648 \mathrm{H}_{2} \mathrm{O}$.

$0,2405 \mathrm{~g} \quad$ " $43,2 \mathrm{ccm}$ Stickgas bei $20^{\circ}$ und $762 \mathrm{~mm}$ Druck.

11) Vergl. Wallach, Amylennitrosat, diese Annalen 241, 288; 248, 161. 


$\begin{array}{lcr} & \text { Berechnet für } & \text { Gefunden } \\ & \mathrm{C}_{6} \mathrm{H}_{9} \mathrm{~N}_{3} \mathrm{O}_{5} & \\ \mathrm{C} & 35,47 & 35,79 \\ \mathrm{H} & 4,43 & 4,83 \\ \mathrm{~N} & 20,63 & 20,74\end{array}$

Die Ueberführung dieses Nitrosats in Ketotrimethyldihydroisoxazol gelingt am besten durch Erhitzen mit der gleichen Gewichtsmenge Essigsäureanhydrid unter Rückfluss auf dem Wasserbade. Die Reaction ist beendet, wenn die Entwickelung brauner Dämpfe aufgehört hat. Man zerstört das Essigsäureanhydrid mit Natronlauge, äthert aus und fractionirt den nach dem Verdampfen des Acthers hinterbleibenden Rückstand im Vacuum. Der Körper siedet unter $16 \mathrm{~mm}$ Druck bei $50-51^{0}$ unter gewöhnlichem Druck bei $151^{\circ}$ und bildet ein stark lichtbrechendes, farbloses Oel von angenehm süsslichem Geruch. Es ist in Wasser unlöslich, von schwach basischen Eigenschaften und besitzt das spec. Gew. 1,020 bei $27^{\circ}$. Ausbeute annähernd quantitativ.

$0,2500 \mathrm{~g}$, Siedep. $50--51^{0}$ bei $16 \mathrm{~mm}$ Druck, gaben $0,5150 \mathrm{CO}_{2}$ und $0,1627 \mathrm{H}_{2} \mathrm{O}$.

$0,1231 \mathrm{~g}$ gaben $11,8 \mathrm{~cm}$ Stickgas bei $20^{\circ}$ und $766 \mathrm{~mm}$ Druck.

$\begin{array}{ccr} & \text { Berechnet für } & \text { Gefunden } \\ & \mathrm{C}_{6} \mathrm{H}_{8} \mathrm{NO}_{2} & \\ \mathrm{C} & 56,69 & 56,18 \\ \mathrm{H} & 7,09 & 7,23 \\ \mathrm{~N} & 11,02 & 11,12\end{array}$

Die Ketoneigenschaften des Productes werden dadurch angezeigt, dass es einerseits nicht Fehling'sche Lösung reducirt und nicht fuchsinschweflige Säure röthet, andererseits ein Oxim (das Isomesitylnitrimin) und ein Phenylhydrazon bildet.

Die cyklische Natur desselben geht einmal aus dem Siedepunkte, sodann aus dem Verhalten gegen Brom und unterbromige Säure hervor. Es addirt nicht Brom und beim Behandeln mit Brom und Natronlauge wird kein Bromoform gebildet.

Mit Hydroxylaminchlorhydrat und Soda in wässrig-alkoholischer Lösung entsteht nach eintägigem Stehen das schön 
krystallisirende Oxim des Trimethyldihydroisoxazols, welches bei $156-157^{0}$ schmilzt und mit dem oben beschriebenen Isomesitylnitrimin identisch ist. Durch Reduction dieses Oxims mit Natrium und Alkohol entsteht eine unangenehm isoxazolartig riechende Base, vielleicht Aminotrimethyldihydroisoxazol, die aber noch nicht näher untersucht wurde.

Das Phenylhydrazon des Trimethyldihydroisoxazols,<smiles>CCNN=C(C)C(C)(C)CC</smiles>

wird erhalten, wenn man äquivalente Mengen essigsaures Phenylhydrazin und Keton in alkoholischer Lösung vermischt und einige Minuten auf $80^{\circ}$ erwärmt. Es krystallisirt aus Alkohol oder Ligroïn in gelben Nadeln vom Schmelzp. 140-141\%.

$0,1321 \mathrm{~g}$, im Vacuum getrocknet, gaben 0,3203 $\mathrm{CO}_{2}$ und $0,0864 \mathrm{H}_{2} \mathrm{O}$. $0,1607 \mathrm{~g}$ gaben $27,1 \mathrm{cem}$ Stickgas bei $21^{\circ}$ und $762 \mathrm{~mm}$ Druck.

$\begin{array}{ccr} & \text { Berechnet fïr } & \text { Gefunden } \\ & \mathrm{C}_{\mathbf{1 2}} \mathrm{H}_{\mathbf{1 5}} \mathrm{N}_{3 \mathbf{3}} \mathrm{O} & \\ \mathrm{C} & 66,36 & 66,28 \\ \mathrm{H} & 6,91 & 7,27 \\ \mathrm{~N} & 19,35 & 19,38\end{array}$

Aus dem Nitrosat kann man auch das Keton bereiten, indem man auf dem Wasserbade so lange erhitzt, als noch rothe Dämpfe entweichen oder durch Behandlung mit der auf ein Mol. Salpetersüure berechneten Menge Natriummethylat. Im letzteren Falle wird $\mathrm{zu} 20,3 \mathrm{~g}$ Nitrosat in $50 \mathrm{ccm}$ Methylalkohol eine Lösung von $4,6 \mathrm{~g}$ Natrium in $100 \mathrm{ccm}$ Methylalkohol gegeben. Das Gemisch wird zum Sieden erhitzt und reagirt dann von selbst ohne Wärmezufuhr weiter. Vom reichlich sich abscheidenden Natriumnitrat wird filtrirt, der Methylalkohol möglichst vollständig im Vacuum abdestillirt, der Rückstand mit Wasser gewaschen und ausgeäthert. Die weitere Behandlung ergiebt sich aus dem früher Gesagten. Nach beiden Methoden ist die Ausbeute nicht so gut, wie beim Erhitzen mit Essigsäureanhydrid. 


\section{Einwirkung der Salpetersäure auf Mesityloxydoxim.}

Erwärmt man vorsichtig eine Lösung ron Mesityloxydoxim (ein Vol.) in verdünnter Salpetersäure vom spec. Gew. 1,1 (sieben Vol.) ${ }^{12}$ ), so beginnen sich nach kurzer Zeit lebhaft braune Dämpfe zu entwickeln. Bei dem Punkte heftigster Reaction inhibirt man sie durch schleuniges Einstellen des Reactionsgefässes in Kältemischung. Diese Operation lässt sich nicht genauer beschreiben, ihr Erfolg ist lediglich Sache der Beobachtung. Beim Ablühlen scheidet sich zunächst ein dickes, gelbes Oel $a b$, welches nach kurzer Zeit theilweise in grossen, gelben Blättern erstarrt. Dieselben lassen sich durch wenig Aether von obigen Beimengungen befreien und können aus Methylalkohol, Aceton, Eisessig oder am besten aus Essigsäureanhydrid umkrystallisirt werden. Der Körper bildet dann grosse, goldgelbe Blätter oder Nadeln, welche bei 128--1290 unter Zersetzung schmelzen. In Aether, Benzol, Petroläther, Wasser ist er schwer löslich.

Die Ausbeute nach dieser Methode ist wechselnd, überschreitet aber selten $40 \mathrm{pC}$, das als Nebenproduct sich bildende gelbe Oel ist noch nicht näher untersucht worden, es ist aber nicht identisch mit dem öligen Nitrosat aus Isomesitylnitrimin. Die Verbindung besitzt die Formel $\mathrm{C}_{6} \mathrm{H}_{3} \mathrm{~N}_{3} \mathrm{O}_{4}$, Analysen und Molekulargewichtsbestimmung sind bereits früher mitgetheilt worden. Zur Bereitung derselben ist das Vorhandensein von salpetriger Säure nothwendig, es geht dies aus einer anderen Darstellungsweise hervor. Man erbält den Körper nämlich viel glatter aus dem Mesitylnitrimin nach folgendem Verfahren:

20 g gut gepulvertes Nitrimin werden mit $80 \mathrm{ccm}$ Salpetersäure rom spec. Gew. 1,1 in einer Porzellanschale übergossen und durch Erwärmen auf dem Wasserbade unter ständigem Umrühren gelöst. Bei vorsichtig geleiteter Operation tritt nur ganz geringe Entwickelung von rothen Dämpfen ein. Wenn

12) Harries, Ber. d. dentsch. chem. Ges. 33, 1999 (1900), vorl. Nittheilung. 
die rothe Flüssigkeit nach einiger Zeit rothe Krystalle abzuscheiden beginnt, hört man mit dem Erwärmen auf und stellt die Schale in Kältemischung, wobei der ganze Inhalt, zumal beim Durchrühren, zu einem dicken, gelbrothen Krystallbrei erstarrt. Derselbe wird auf einer Nutsche abgepresst, zuerst mit Wasser, dann mit einem Gemenge von wenig kaltem Methylalkohol und Aether, zuletzt mit Aether allein gewaschen. Die so behandelte Substanz ist beinahe analysenrein, die Ausbeute davon beträgt aus $20 \mathrm{~g}$ Nitrimin $19 \mathrm{~g}$. Sic ist identisch mit dem vorhin beschriebenen Producte der directen Oxydation des Mesityloxydoxims durch Salpetersäure. Für die präparative Bereitung empfiehlt sich nur diese Methode.

Der neue Körper zeigt auf Lackmus saure Reaction und ist gegen Alkalien äusserst empfindlich, beim Uebergiessen mit Natronlauge zersetzt er sich bisweilen mit solcher Heftigkeit, dass Feuererscheinung auftritt, mit organischen Basen entwickelt er ein Gas, wahrscheinlich Stickoxydul. Dagegen verbält er sich äusserst beständig gegen Säuren, aus rauchender Salzsäure und concentrirter Salpetersäure kann man ihn umkrystallisiren, auch von Brom bleibt er nnangegriffen. Durch Einleiten von Bromwasserstoff in eine Chloroformlösung der Verbindung erhält man nach dem Verdunsten des Chloroforms eine hellgelbe Substanz, die die frormel $\mathrm{C}_{6} \mathrm{H}_{9} \mathrm{~N}_{2} \mathrm{O}_{2} \mathrm{Br}$ zu besitzen scheint, also die Gruppe $\mathrm{NO}_{2}=0 \mathrm{~N} . \mathrm{O}$ gegen $\mathrm{Br}$ ausgetauscht hat.

\section{Constitution des Oxydationsproductes des Mesityloxydoxims.}

Ich habe in der scbon erwähnten vorläufigen Mittheilung zwei Constitntionsformeln für das Oxydationsproduct aufgestellt, indem als Zwischenproducte zunächst Anlagerung der salpetrigen oder Salpetersäure an die doppelte Bindung und darauffolgende Oxydation des mittelständigen Kohlenstoffatoms zur Oximidogruppe angenommen wurden ${ }^{13}$ ).

13) Is findet sich in der dortigen Fntwickelnng ein Fehlor, der hier berichtigt sein möge. 
244 Harries, Ueber die Einwirkung der salpetrigen und der

I.<smiles>CC(CC(C)(C)O)=[N+]([O-])O</smiles><smiles>CC(=O)C(=NO)C(C)(C)C</smiles>

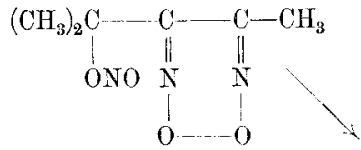
$\mathrm{C}_{6} \mathrm{H}_{9} \mathrm{~N}_{8} \mathrm{O}_{4}$
II.<smiles>CC(C)(C)CC(C)(C)C</smiles>
$\mathrm{ONO}_{2}$ NOH

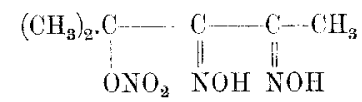<smiles>Cc1nonc1C(C)(C)O[Na]</smiles>

Eine dritte Erklärung, dass ein Nitromesitylnitrimin der Formel<smiles>CC(C)=C(C(C)C)[N+](=O)[O-]</smiles>

vorliege, ist wegen der gesättigten Natur der Verbindung nicht haltbar.

Im Fall I wäre ein Nitritmesityldioximperoxyd, im Fall II ein Nitrosatmesityifurazan entstanden.

Wie ich gleich bemerken will, scheint mir jetzt die in I gegebene Constitution in etwas modificirter Form den Umwandlungen, welche beobachtet worden sind, zu genügen, denn es ist nicht gelungen, durch Natriumalkoholat Natriumnitrat in glatter Weise abzuspalten und somit die Nitrosatgruppe nachzuweisen.

Die Verbindung enthält jedenfalls eine Nitrit- und eine Dioximperoxydgruppe. Die Dioximperoxyd- oder Glyoximhyperoxydgruppe ist schon in einer ganzen Reihe von Körpern, die mit den hier geschilderten nahe verwandt erscheinen, angenommen worden. So zuerst von Hollemann ${ }^{14}$ ) in dem Einwirkungsproducte von Salpetersäure vom spec. Gew. 1,4 auf Acetophenon

14) Ber. d. deutsch. chem. Ges. 20, 3359 (1887); 21, 2835 (1898); Ree. d. B. 11, 268 (1892). 


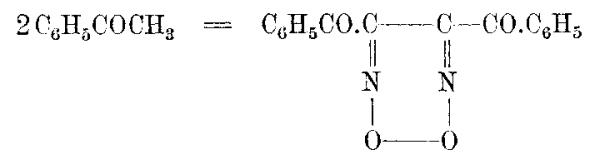

dem Diphenyldinitrosacyl, nachdem schon vorher Koreff ${ }^{15}$ ) und Ilinski ${ }^{16}$ ) gezeigt hatten, dass Naphtochinondioxim durch oxydirende Mittel in eine um zwei Wasserstoffatome ärmere Verbindung übergehe.

Von Scholl ${ }^{17}$ ) wurde die Einwirkung von Stickstofftetroxyd auf Dioxime untersucht und festgestellt, dass diese ganz allgemein die Fähigkeit besitzen, sich dadurch zu Glyoximhyperoxyden oxydiren zu lassen:<smiles>[Y10]C1=NOON=C1[R]</smiles>

Der Peroxyddiisonitrosobuttersäureester wurde von Angeli ${ }^{18}$ ) und Jowitschitsch ${ }^{19}$ ), der Peroxyddisonitrosobernsteinester von Proepper ${ }^{20}$ ) und $B \mathrm{eckh}^{21}$ ) dargestellt:<smiles></smiles><smiles>CCOC(=O)C1=NOO[Y]=C1C(=O)OC</smiles>

Hervorzuheben sind hier die bewunderwogswürdig durchgearbeiteten Untersuchungen von Behrend ${ }^{22}$ ) und seinen Schülern ïber die Einwirkungsproducte der Salpetersäure auf Aceton.

Dabei wurde die Acetylmethylnitrolsäure erhalten, welche durch Hydroxylamin in Isonitrosonitroacetoxim und durch sal-

\footnotetext{
15) Ber. d. deutsch. chem. Ges. 19, 181 (1886).

16) Ber. a. dentsch. chem. Ges. 19, 349 (1886).

$\left.{ }^{17}\right)$ Ber. d. dentsch, chem. Ges. $\mathbf{2 3}, 3496$ (1890).

18) Ber. d. deutsch. chem. Ges. :6, ๖94 (1893).

19) Ber. d. deutsch. chem. Ges. 28, 2096, 2679 (1895).

20) Diese Annalen $\mathbf{2 2 2}, 46$ (1883).

$\left.{ }^{21}\right)$ Ber. d. dentsch. chem. Ges. BU, 152 (1897).
}

22) Diese Amalen a77, 317 (1893); 283, 222 (1894); 309, 242 (1900). 
petersalpetrige Säure in Nitromethylglyoximhyperoxyd umgewandelt werden konnte:

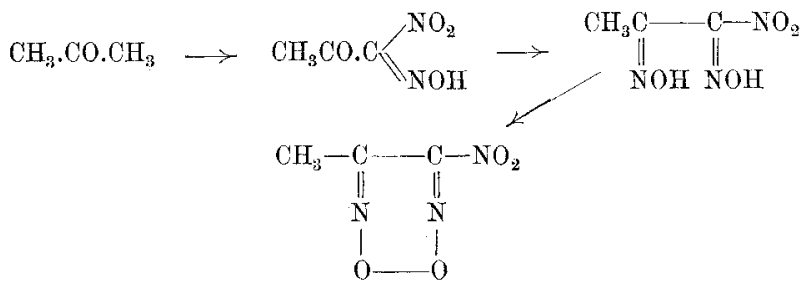

Man kann sagen, dass ich gewissermassen den umgekehrten Weg eingeschlagen habe, Behrend oxydirt zuerst das Keton mit Salpetersäure und behandelt das entstandene Product mit Iydroxylamin, während ich erst oximire und dann mit Salpetersäure oxydire. Aus Gründen der Analogie wird man wohl annehmen dürfen, dass in dem Oxydationsproducte des Mesityloxydoxims die Glyoximhyperoxydgruppe vorhanden ist, dann bleibt für den Restcomplex $\mathrm{NO}_{2}$ nur die Formulirung als Nitritgruppe übrig.

Bei Annahme der Nitritglyoximhyperoxydformel sind aber immer noch zwei Möglichkeiten für die Constitution dieses Körpers gegeben:

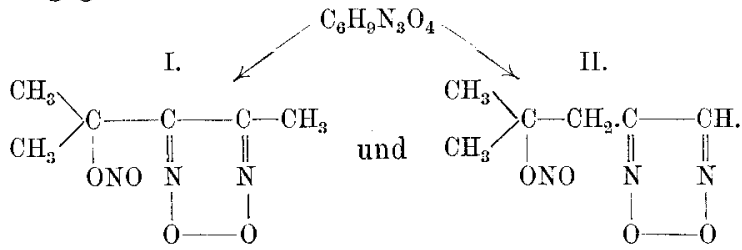

Ich habe mich für die Formel II entschieden, obgleich sie zuerst etwas frappirend wirkt, weil das aus dem Hyperoxyd durch Anilin entstehende Nitromesityloxyd die Nitrogruppe an einem primären und nicht an einem tertiären Kohlenstoffatom, wie nach Formel I zu erwarten wäre, enthält:<smiles>CC(=O)C(=C(C)C)[N+](=O)[O-]</smiles>

tertiäres Nitromesityloxyd<smiles>CC(C)=CC(=O)C[O+]=O</smiles>

primäres Nitromesity]oxyd. 
Dasselbe condensirt sich nämlich nicht mit Benzaldehyd wie das Nitroaceton selbst und bildet ein schwerlösliches Natriumsalz, Beobachtungen, welche nur mit einem primären Nitromesityloxyd in Einklang zu bringen sind. Ich bezeichne den Oxydationskörper von nun an mit dem Namen Nitritmesitylglyoximhyperoxyd.

Einwirkung von Anitin auf das Nitritmesitylglyoximhyperoxyd.

Holleman ${ }^{23}$ ) hat bereits beim Diphenyldinitrosacyl beobachtet, dass sich die Glyoximhyperoxydgruppe durch Anilin zersetzen lässt, er fand neben Benzanilid einen Körper, über dessen Constitution er nur Vermuthungen anstellt.

Ich habe gefunden, dass das Nitritmesitylglyoximhyperoxyd sich verschieden gegenüber Anilin verhält, je nachdem man es in ätherischer oder Eisessiglösung darauf einwirken lässt. In beiden Fällen ist es gleichgültig für die Ausbeute, ob ein oder zwer Mol. Anilin angewendet werden.

\section{Einwirkung von Anilin in ätherischer Lösung auf das}

Nitritmesitylglyoximhyperoxyd, Anil-a-nitromesityloxyd.

$10 \mathrm{~g}$ des Hyperoxyds werden in $50 \mathrm{ccm}$ absolutem Aether suspendirt und $5 \mathrm{~g}$ Anilin in kleinen Portionen dazu gegeben. Der gelbe Körper geht unter lebhafter Gasentwickelung (Stickoxydul) in Lösung. Um die Reaction zu vervollständigen wird zuletzt noch kurze Zeit auf dem Wasserbade erwärmt, von etwas anangegriffener gelber Substanz abfiltrirt und nunmehr der Aether verdampft. Der Rückstand, welcher noch während circa zehn Minuten auf dem Wasserbade erwärmt werden muss, erstarrt nach einiger Zeit beim Reiben zu einem braunen Krystallkuchen. Derselbe wird auf Thon von anhaftenden schmierigen Beimengungen befreit und aus 95 procentigem Alkohol oder Petroläther umkrystallisirt. Das Product, welches man in einer Ausbeute von circa $85 \mathrm{pC}$. der Theorie erhält, schiesst

\footnotetext{
23) loc. cit.
} 
in prächtigen, gelben Blättern an. Es wird mit Ausnahme von Wasser von den organischen Lösungsmitteln leicht aufgenommen und schmilzt scharf bei $84-85^{\circ}$.

I. $0,1786 \mathrm{~g}$, im Vacuum getrocknet, gaben $0,4304 \mathrm{CO}_{2}$ und 0,1049 $\mathrm{H}_{2} \mathrm{O}$.

II. $0,1770 \mathrm{~g}$ gaben $0,4260 \mathrm{CO}_{2}$ und $0,1036 \mathrm{H}_{2} \mathrm{O}$.

III. $0,1934 \mathrm{~g} \quad " 22,0 \mathrm{~cm}$ Stickgas bei $21^{\circ}$ und $764 \mathrm{~mm}$ Druck. VI. $0,2089 \mathrm{~g} \quad, \quad 23,6 \mathrm{ccm} \quad " \quad, 19^{0} \quad, 764 \mathrm{~mm}$ Druck.

Berechnet für $\mathrm{C}_{12} \mathrm{H}_{14} \mathrm{~N}_{2} \mathrm{O}_{2}$

$\mathrm{C}$

$\mathrm{H}$

$\mathrm{N}$

$$
66,05
$$

6,42

12,84

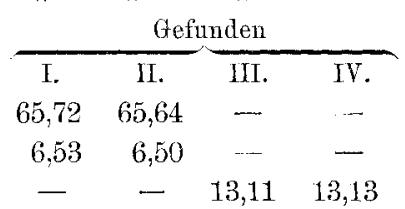

Molekulargewichtsbestimmung in Benzol nach Ra oult-Beckmann. $0,1718 \mathrm{~g}$ gaben in $23,13 \mathrm{~g}$ Benzol eine Depression von $0,179^{\circ}$. Berechnet

Mol.-Gew. 218

Die Einwirkung des Anilins auf das Iyperoxyd in Aether verläuft nach der Gleichung:

$$
\mathrm{C}_{6} \mathrm{H}_{9} \mathrm{~N}_{3} \mathrm{O}_{4}+\mathrm{NH}_{2} \mathrm{C}_{6} \mathrm{H}_{5}=\mathrm{C}_{12} \mathrm{H}_{14} \mathrm{~N}_{2} \mathrm{O}_{3}+\mathrm{N}_{2} \mathrm{O}+\mathrm{H}_{2} \mathrm{O}
$$

und lässt sich in folgendem Schema erklären:

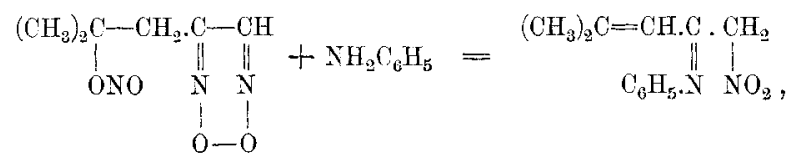

d. h. durch das Anilin wird Salpetrigsäure abgespalten, welche in statu nascendi auf die Hyperoxydgruppe unter Bildung von Stickoxydul und einem Carbonyl einwirkt, während Anilin sich mit der Carbonylgruppe unter Wasseraustritt umsetzt.

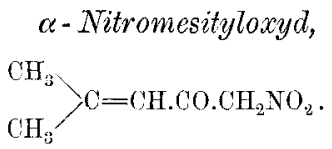

Das Anilnitromesityloxyd kann durch verdünnte Mineralsäuren sehr leicht in Anilin und a-Nitromesityloxyd gespalten werden. $\mathrm{Zu}$ diesem $\mathrm{Zwecke}$ werden $10 \mathrm{~g}$ Anilverbindung mit 
$30 \mathrm{ccm}$ concentrirter Salzsäure übergossen und so lange ohne Erwärmen damit digerirt, bis sie vollständig gelöst ist. Darauf wird mit $100 \mathrm{ccm}$ Wasser verdünnt und ausgeäthert. Die ätherische Lösung wird mit Soda von Salzsäure befreit und das nach dem Verdampfen des Aethers zuräckbleibende Oel mit Natriumsulfat getrocknet. Im Vacuum fractionirt siedet es bei $95-96^{\circ}$ unter $23 \mathrm{~mm}$ Druck und bildet ein gelbes Liquidum von stechendem, wenig an Mesityloxyd erinnerndem Geruch. Das specifische Gewicht beträgt bei $27,3^{0}$ 1,212. In kleinen Portionen siedet es bei gewöhnlichem Druck unzersetzt.

$0,2049 \mathrm{~g}$ gaben $0,3736 \mathrm{CO}_{2}$ and $0,1179 \mathrm{H}_{2} \mathrm{O}$.

$0,2045 \mathrm{~g} \quad " \quad 17,9 \mathrm{ccm}$ Stickgas bei $23^{\circ}$ und $764 \mathrm{~mm}$ Druck.

Berechnet für.

$\mathrm{C}_{6} \mathrm{H}_{9} \mathrm{NO}_{3}$

C

H

$\mathrm{N}$
50,35

6,29

9,79
Gefunden

49,73

6,39

9,99

Molekulargewichtsbestimmung in Benzol nach Ra on t-Beckmann.

$0,1413 \mathrm{~g}$ gaben in $21 \mathrm{~g}$ Benzol eine Depression von $0,240^{\circ}$.

$\begin{array}{ccc} & \text { Bereclinet } & \text { Gefunden } \\ \text { Nolgew. } & 143 & 140\end{array}$

Dass in dem beschriebenen Körper $\mathrm{C}_{6} \mathrm{H}_{9} \mathrm{NO}_{3}$ ein Nitromesityloxyd vorliegt, muss aus folgenden Reactionen geschlossen werden. Die Verbindung ist ungesättigt, da sie momentan Brom in Schwefelkohlenstoff entfärbt und leicht unter verschiedenen Bedingungen in das von $\mathrm{Lucas}$ dargestellte Nitroaceton und Aceton zerfällt. So erhält man bei der Einwirkung von Semicarbazid nach der Thiele-Baeyer'schen Methode nicht das Semicarbazon des Körpers $\mathrm{C}_{6} \mathrm{H}_{9} \mathrm{NO}_{3}$, sondern gleich unter Spaltung dasjenige des Nitroaceton. Ebenso liefert Anilin und Eisessig das Anilnitroaceton, beide Derivate sind später beschrieben worden. Die Nitrogruppe haftete an einem primären Kohlenstoffatome, da sich beim Uebergiessen mit Natronlauge sofort ein schön krystallisirendes Natriumsalz ausscheidet, aus welchem verdünnte Schwefelsäure wieder das Nitromesityloxyd 
regenerirt. Von verdünnter Natronlauge oder Soda wird es schwer aufgenommen, da es in Wasser unlöslich ist. Beim Stehen mit Benzaldehyd und Natronlauge tritt keine Condensation ein, woraus ebenfalls hervorgeht, dass diese Nitroverbindung nicht die Acetylgruppe $-\mathrm{CO}^{-\mathrm{CH}_{3}}$ enthält.

\section{Kinwirkung von Anilin in Eisessig auf das Nitrit-} mesitylglyoximhyperoxyd.

\section{Anilnitroaceton,}<smiles>CC(=NC(=O)c1ccccc1)C(C)CC(=O)O</smiles>

$10 \mathrm{~g}$ des Körpers $\mathrm{C}_{6} \mathrm{H}_{9} \mathrm{~N}_{3} \mathrm{O}_{4}$ werden mit $30 \mathrm{ccm}$ Eisessig überschichtet und dazu 5 g Anilin gegeben. Unter lebbafter Gasentwickelung geht die gelbe Verbindung in Lösung. Darauf wird der Eisessig im Vacuum abgedampft, wobei ein dickes, dunkelbrames Oel hinterbleibt. Dasselbe lässt sich durch Verrühren mit wenig Wasser leicht zur Krystallisation bringen. Die Krystalle werden abgesaugt, auf Thon gestrichen und aus Alkohol oder Ligroïn umkrystallisirt. Sie bilden schöne schwach gelbe Nadeln, welche bei $80^{\circ} \mathrm{zu}$ sintern anfangen und bei $87^{\circ}$ schmelzen. Beim langsamen Abdunsten des Lösungsmittels werden dicke, bernsteingelbe, schiefe Prismen gewonnen. Die Ausbeute beträgt etwa 90 pC. der Theorie.

$0,1826 \mathrm{~g}$ gaben $0,4047 \mathrm{CO}_{2}$ und $0,0933 \mathrm{H}_{2} \mathrm{O}$.

$0,1441 \mathrm{~g} \quad " 20,0 \mathrm{~cm}$ Stickgas bei $20^{\circ}$ und $750 \mathrm{~mm}$ Druck.

Berechnet für $\mathrm{C}_{9} \mathrm{H}_{10} \mathrm{~N}_{9} \mathrm{O}_{2}$

$\mathrm{C}$

H

$\mathrm{N}$
60,67

5,62

15,73
Gefunden.

60,45

5,68

15,77

Nolekulargewichtsbestimmung in Benzol nach Raoult-Beckmaun.

$0,1648 \mathrm{~g}$ gaben in 20,55 $\mathrm{g}$ Benzol eine Depression von $0,228^{\circ}$.

Berechnet

Mol.-Gew.
Gefunden

176 
Aus ihrem Verhalten gegen verdünnte Schwefelsäure geht die Natur der Verbindung als Anilderivat des Nitroacetons hervor. Hierbei wird sie quantitativ gespalten in Anilin und Nitroaceton: $\mathrm{C}_{9} \mathrm{H}_{10} \mathrm{~N}_{2} \mathrm{O}_{2}+\mathrm{H}_{2} \mathrm{O}=\mathrm{C}_{6} \mathrm{H}_{5} \cdot \mathrm{NH}_{2}+\mathrm{CH}_{3} \mathrm{COCH}_{2} \mathrm{NO}_{2}$.

Nitroaceton, $\mathrm{CH}_{3} \mathrm{CO} . \mathrm{CH}_{2} \mathrm{NO}_{2}$.

$10 \mathrm{~g}$ der Anilverbindung werden mit $50 \mathrm{ccm} 25$ procentiger Schwefelsäure übergossen und so lange auf dem Wasserbade digerirt, bis vollständige Lösung erfolgt ist. Das Reactionsgegemisch wird fünf bis sechs Mal mit Aether ausgeschüttelt. Nach dem Verdunsten desselben hinterbleibt eine schwach röthlich gefärbte Krystallmasse, welche aus absolutem Aether oder absolutem Alkohol in rein weissen, derben Nadeln oder grossen Säulen anschiesst, welche bei $49-50^{\circ}$ schmelzen. Die Ausbeute ist quantitativ.

$0,1451 \mathrm{~g}$, im Vacuum getrocknet, gaben $0,1853 \mathrm{CO}_{2}$ und $0,0662 \mathrm{H}_{2} \mathrm{O}$. $0,1541 \mathrm{~g}$ gaben $18,4 \mathrm{ccm}$ Stickgas bei $23^{\circ}$ und $767 \mathrm{~mm}$ Druck.

$\begin{array}{ccr} & \text { Berechnet für } & \text { Gofunden } \\ & \mathrm{C}_{3} \mathrm{H}_{5} \mathrm{NO}_{3} & \\ \mathrm{C} & 34,95 & 34,83 \\ \mathrm{H} & 4,85 & 5,07 \\ \mathrm{~N} & 13,59 & 13,69\end{array}$

Molekulargewichtsbestimmung in Benzol nach Raoult-Beckmann.

$0,1539 \mathrm{~g}$ gaben in $17,53 \mathrm{~g}$ Benzol eine Depression von $0,415^{\circ}$.

$\begin{array}{ccc} & \text { Berechnet } & \text { Gofunden } \\ \text { Mol.-Gew. } & 103 & 106\end{array}$

Die hier beschriebene Verbindung ist in jeder Beziehung identisch mit dem von A. Lucas ${ }^{24}$ ) aus Jodaceton und Silbernitrit bereiteten Nitroaceton, dessen Constitution unzweideutig durch Reduction zum Aminoaceton bewiesen wurde. Das Verfahren zur Darstellung, welches ich gegeben habe, scheint mir aber, namentlich bei Bedarf grösserer Mengen, ungleich brauchbarer zu sein. Man erhält bei durchschnittlichen Ausbeuten

$\left.{ }^{24}\right)$ Ber. d. deutsch. chem. Ges. a2, 604, 3179 (1899). 
aus $100 \mathrm{~g}$ Mesityloxyd circa $70 \mathrm{~g} \alpha$-Mesityloxim und daraus etwa $35 \mathrm{~g}$ reines Nitroaceton.

Da mir eine reichliche Menge Nitroaceton zu Gebote stand, habe ich dasselbe noch etwas eingehender untersucht und kann Folgendes zur Charakteristik nachtragen.

Das Nitroaceton siedet bei raschem Erhitzen in kleinen Portionen nur unter geringer Zersetzung gegen 185- $190^{\circ}$, unter $24 \mathrm{~mm}$ Druck unzersetzt bei $103-104^{\circ}$. Beim mehrtägigen Aufbewahren oder beim langsamen Destilliren grösserer Mengen aus dem Oelbade bei $165^{\circ}$ zersetzt es sich unter Bildung von Essigsäure und einer stechenden, nach Blausäure riechenden Substanz, wahrscheinlich Cyansäure, wie durch folgende Gleichung ausgedrückt wird:

$$
\mathrm{CH}_{3} \mathrm{COCH}_{2} \mathrm{NO}_{2}=\mathrm{CH}_{3} \mathrm{COOH}+\mathrm{CNOH} \text {. }
$$

Der Körper löst sich in den meisten Lösungsmitteln sehr leicht, die wässrige Lösung reagirt sauer und giebt mit Eisenchlorid keine Färbung. Er ist mit Wasserdampf leicht flüchtig. Von seinen Salzen sind ausser dem von Lucas analysirten Ammoniumsalz noch das Natrium- und das Silbersalz zu erwähnen. Das Natriumsalz bildet sich beim Uebergiessen des Nitroacetons mit concentrirter Natronlauge in farblosen Krystallen von der Zusammensetzung $\mathrm{CH}_{3} \mathrm{COCH}=\mathrm{NO}_{2} \mathrm{Na}$.

$0,1567 \mathrm{~g}$ gaben $0,0864 \mathrm{Na}_{2} \mathrm{SO}_{4}$.

$$
\begin{gathered}
\text { Berechnet für } \\
\mathrm{C}_{3} \mathrm{H}_{4} \mathrm{O}_{3} \mathrm{Na}
\end{gathered}
$$

$\mathrm{Na} \quad 18,4$

17,86

Das Silbersalz entsteht nach dem Neutralisiren mit Ammoniak durch Fällen mit Silbernitrat und bildet schöne gelbe Nadeln, welche sich in viel Wasser lösen und dabei theilweise zersetzen. Am Lichte wird die Verbindung bald schwarz.

Das Nitroaceton reducirt beim Kochen nicht ammoniakalische Silberlösung. 
Nitroacetonsemicarbazon,

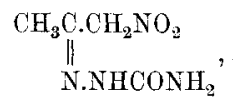

entsteht leicht wach der Thiele-Baeyer'schen Methode, krystallisirt in Nadeln und schmilzt nach dem Umkrystallisiren aus Alkohol bei $163-164^{\circ}$. Dieselbe Verbindung wird auch aus Nitromesityloxyd gewonnen.

$0,167 \mathrm{~g}$ gaben $51,4 \mathrm{ccm}$ Stickgas bei $23^{\circ}$ und $759 \mathrm{~mm}$ Druck.

$\begin{array}{ccc} & \text { Berechnet fuir } & \text { Gefunden } \\ & \mathrm{C}_{4} \mathrm{H}_{8} \mathrm{~N}_{4} \mathrm{O}_{3} & \\ \mathrm{~N} & 35,00 & 34,98\end{array}$

Das zunächst immer als Oel sich abscheidende Phenylhydrazon ist bereits von Lucas untersucht worden.

Auch ich konnte wie Lucas nach dem gewöhnlichen Verfahren kein Oxim des Nitroacetons gewinnen. Ich mache aber darauf aufmerksam, dass das Oxim des Nitroacetons schon früher von Jowitschitsch ${ }^{25}$ ) beschrieben worden ist, welcher es durch Spaltung des Nitromethylisoxalous vermittelst Alkali bereitete:

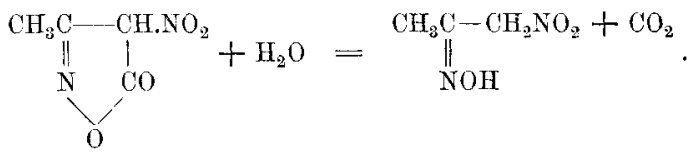

Diese Verbindung wird sich daher wohl noch auf dem einen oder anderen Wege auch aus dem Nitroaceton selbst erhalten lassen. Fügt man das Nitroaceton zu einer wässrigen Lösung von Natriumnitrit, so findet angenblicklich Umsetzung statt, wobei die Flüssigkeit eine gelbliche Trübung annimmt.

Das Reactionsproduct kann durch Aether isolirt werden und bildet ein die Haut stark ätzendes Product, jedenfalls die Acetylmethylnitrolsäure von Behrend und Tryller ${ }^{26}$ ), welche dieselbe umgekehrt aus Isonitrosoaceton und rauchender Sal-

$\left.{ }^{25}\right)$ Ber. d. deutsch. chem. Ges. 28, 2100 (1895).

26) Diese Annalen 283, 222 (1894). 
petersäure bereitet haben. Dagegen reagirt das Nitroaceton nicht mit Nitrosodimethylanilin, wie mir Herr Dr. Sachs die Güte hatte mitzutheilen.

Das charakteristischste Derivat des Nitroacetons ist seine Anilverbindung, die ich für seinen Nachweis am geeignetsten halte. Ich wundere mich, dass Lucas dieselbe nicht schon entdeckt hat.

Zu ihrer Darstellung löst man $2 \mathrm{~g}$ Nitroaceton in $15 \mathrm{ccm}$ 50 procentiger Essigsäure, fügt $2 \mathrm{~g}$ Anilin hinzu und erwärmt schwach auf dem Wasserbade. Beim Anspritzen mit einigen Tropfen Wasser krystallisirt die prächtige Substanz in gelben Nadeln herans, welche aus absolutem Alkohol umkrystallisirt bei $87^{\circ}$ schmelzen und mit dem früher beschriebenen Körper $\mathrm{C}_{9} \mathrm{H}_{10} \mathrm{~N}_{2} \mathrm{O}_{3}$ identisch sind. Zum genauen Vergleich wurde noch eine Elementaranalyse ausgeführt.

$0,1743 \mathrm{~g}$, vacuumtrocken, gaben $0,3861 \mathrm{CO}_{2}$ und $0,0907 \mathrm{H}_{2} \mathrm{O}$.

$0,1440 \mathrm{~g}$ gaben $19,8 \mathrm{ccm}$ Stjekgas bei $23^{\circ}$ und $765 \mathrm{~mm}$ Druck.

$\begin{array}{ccr} & \text { Berechnet für } & \text { Gefunden } \\ & \mathrm{C}_{9} \mathrm{H}_{10} \mathrm{~N}_{2} \mathrm{O}_{3} & \\ \mathrm{C} & 60,67 & 60,41 \\ \mathrm{H} & \overline{0}, 62 & 5,78 \\ \mathrm{~N} & 15,73 & 15,72\end{array}$

a-Nitrobenzalaceton, $\mathrm{C}_{6} \mathrm{H}_{5} \mathrm{CH}=\mathrm{CH} . \mathrm{CO} . \mathrm{CH}_{2} \mathrm{NO}_{2}$.

Wenn man die Lösung von $1 \mathrm{~g}$ Nitroaceton in $10 \mathrm{ccm}$ fünfprocentiger Natronlauge mit $1 \mathrm{~g}$ Benzaldehyd bis zur bleibenden Emulsion schüttelt, so ist nach 24 stündigem Stehen die ganze Masse zu einem dicken Brei erstarrt. Das Condensationsproduct ist in diesem Zustande ein schwer lösliches Natriumsalz, aus welchem mit verdünnter Mineralsäure das Nitrobenzalaceton in Freiheit gesetzt wird. Dasselbe krystallisirt aus absolutem Alkohol in schwach gelb gefärbten Tafeln, die meistens die Form von Trapezen besitzen und häufig zu Zwillingen vereinigt sind. Sie sintern bei $83^{\circ}$ und schmelzen bei $87-88^{\circ}$ vollständig; die Ausbente ist quantitativ. Die Substanz wird von Wasser nicht, von verdünntem Alkali in der 
Kälte schwer, in der Wärme leicht mit gelber Farbe aufgenommen.

$0,1.543 \mathrm{~g}$, vacuumtrocken, gaben $0,3564 \mathrm{CO}_{2}$ und $0,0693 \mathrm{H}_{2} \mathrm{O}$. $0,1645 \mathrm{~g}$ gaben $11 \mathrm{ccm}$ Stickgas bei $24^{\circ}$ und $759 \mathrm{~mm}$ Druck.

$\begin{array}{ccr} & \text { Berechnet für } & \text { Gefunden } \\ & \mathrm{C}_{10} \mathrm{H}_{9} \mathrm{NO}_{3} & \\ \mathrm{C} & 62,83 & 62,99 \\ \mathrm{H} & 4,71 & 4,99 \\ \mathrm{~N} & 7,33 & 7,55\end{array}$

Durch Anilin und Eisessig wird das Nitrobenzalaceton nicht wie das Nitromesityloxyd gespalten, es bleibt unverändert und scheint keine Anilverbindung zu liefern.

Zum Schlusse möchte ich noch eine kurze Bemerkung über das ölige Nitroaceton, welches Henry und de Baltice ${ }^{27}$ ) bekanntlich ans Nitroisopropylalkohol durch Oxydation erhalten haben wollen, anknüpfen. Ich nahm, um diesen Punkt aufzuklären, mit dem krystallisirten Nitroaceton verschiedenartige Umlagerungsversuche vor, doch ist es mir unmöglich gewesen, das ölige Nitroaceton der genannten Forscher zu erhalten. Im Hinblick auf eine neuere Reclamation von Henry ${ }^{28}$ ) glaube ich mein Resultat nicht zurückhalten zu dürfen und schliesse mich im Uebrigen ganz den Ausführungen der Herren Lucas und Hantzsch${ }^{29}$ ) an.

Bei der experimentellen Untersuchung bin ich von Herrn Dr. Richard Gley unterstützt worden, wofür ich demselben meinen Dank ausspreche.

Nachschrift. Ich weise noch darauf hin, dass meiner Meinung nach in den Reactionen des Nitritmesitylglyoximhyperoxyds einerseits und des Terpinennitrits und Phellandrennitrits andererseits eine gewisse Analogie besteht. Letztere gehen

27) Chem. Centralb1. 1899, I, 1020.

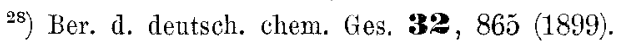

29) 1oc. cit. 
256 Harries, Ueber die Einwirkung der salpetrigen etc.

bei der Behandlung mit Alkalien in sogenannte Nitrosooxyde (Wallach $\left.{ }^{30}\right)$ ) oder Oxyoxime (Semmler $\left.{ }^{31}\right)$ ) über. Mir scheint nun, dass das Phellandrennitrosooxyd (Nitrophellandren Pesci's) und das Terpinenoxyoxim dem $\alpha$-Nitromesityloxyd entsprechen und als Pseudonitrokörper anzusehen sind. Diese Auffassung würde ibre Reducirbarkeit zu Ketonen und Aminbasen sehr wohl erklären, da Konowal off ${ }^{32}$ ) und Markowniko ff ${ }^{33}$ ) in einer Reihe von Beispielen gezeigt haben, dass die Nitroderivate des Cyklohexans zu Ketoneu und Basen reducirt werden können.

30) Wa 11 ach, diese Annalen $\mathbf{3 1 3}, 345,361$ (1900).

31) Semmler, Ber. d. deutsch. chem. Ges. 34, 710 (1901).

32) Konowaloff, Chem. Centralbl. 1899, I, 597.

33) Markownik off, diese Annalen 302, 1 (1899).

(Geschlossen am 15. November 1901.) 